\title{
A conjuntura dos conflitos ambientais no Estado do Pará
}

\author{
The situation of environmental conflicts in the State of Pará \\ La situación de los conflictos ambientales en el Estado de Pará
}

Recebido: 23/07/2021 | Revisado: 29/07/2021 | Aceito: 18/09/2021 | Publicado: 19/09/2021

Hellem Cristina Teixeira Rodrigues ORCID: https://orcid.org/0000-0003-2671-8742 Universidade Federal Rural da Amazônia, Brasil E-mail:hellem.cristinat@gmail.com

Isabela Lopes de Oliveira ORCID: https://orcid.org/0000-0002-6909-4303 Universidade Estácio de Sá, Brasil

E-mail: isabela.oliveira.1894@gmail.com

Breno Eduardo dos Santos Alves ORCID: https://orcid.org/0000-0003-4500-821X Universidade Federal Rural da Amazônia, Brasil E-mail: brenoedu_leao@hotmail.com

Erika Souza Guimarães Pacheco ORCID: https://orcid.org/0000-0003-1979-304X Universidade Estácio de Sá, Brasil E-mail: guimaerika@gmail.com

Rhayme Karolinne Vieira Martins ORCID: https://orcid.org/0000-0001-5419-5147 Universidade Estácio de Sá, Brasil E-mail: rhayme_marrtins@hotmail.com

Francimary da Silva Carneiro ORCID: https://orcid.org/0000-0002-1693-8779 Secretaria de Estado de Meio Ambiente e Sustentabilidade, Brasil E-mail: francimarycarneiro@gmail.com

Ana Marcela Alves dos Santos ORCID: https://orcid.org/0000-0002-9498-8587 Secretaria de Estado de Meio Ambiente e Sustentabilidade, Brasil

E-mail: anamarcela-santos@hotmail.com

Carla Vanessa Borges Castro ORCID: https://orcid.org/0000-0002-5066-0862 Secretaria de Estado de Meio Ambiente e Sustentabilidade, Brasil E-mail: carlavbcastro@yahoo.com.br

Marcio Braga Amorim

ORCID: https://orcid.org/0000-0001-7508-2766 Secretaria de Estado de Meio Ambiente e Sustentabilidade, Brasil E-mail: marciobamorim@gmail.com

Klewton Adriano Oliveira Pinheiro

ORCID: https://orcid.org/0000-0003-2696-4249 Instituto Federal de Educação, Ciência e Tecnologia do Pará, Brasil E-mail: klewton.pinheiro@gmail.com

\section{Resumo}

Os conflitos ambientais se tornaram uma realidade mundial, no Brasil especificamente no Estado do Pará, foi palco de grandes histórias entristecedoras por buscarem a preservação dos recursos naturais, apresentando variadas categorias de conflitos, seja pela grande quantidade de matérias-primas ou pelo Arco do desmatamento. O mapeamento destes conflitos é fundamental para a preservação da sadia qualidade de vida, por isso, este trabalho tem o intuito de espacializar áreas de enfrentamentos ambientais do Estado, levando em consideração os dados do Atlas de Justiça Ambiental que contabilizou 24 ocorrência para a área de estudo, somado a dados disponibilizados pelo IBGE, assim como o uso do software Excel e de processamento de dados ArcGIS. Sendo possível espacializar as cidades do Pará com o seu tipo de conflito. Logo, pode-se afirmar a diversidade de conflitos enfrentados no Estado, refletindo ao desafio de lidar e gerir as situações, daí recomenda-se a ampliação deste tipo de estudo, com enfoque na gestão eficiente dos territórios.

Palavras-chave: Monitoramento; Espacialização; Exploração; Meio ambiente.

\section{Abstract}

Environmental conflicts have become a global reality, in Brazil specifically in the State of Pará, it was the scene of great saddening stories for seeking the preservation of natural resources, presenting various categories of conflicts, 
whether due to the large amount of raw materials or the Arco do logging. The mapping of these conflicts is fundamental for the preservation of a healthy quality of life, therefore, this work aims to spatialize areas of environmental confrontations in the State, taking into account the data from the Atlas of Environmental Justice, which counted 24 occurrences for the area of study, added to data provided by IBGE, as well as the use of Excel and ArcGIS data processing software. It is possible to spatialize the cities of Pará with their type of conflict. Therefore, it is possible to affirm the diversity of conflicts faced in the State, reflecting the challenge of dealing and managing situations, hence it is recommended to expand this type of study, focusing on the efficient management of territories.

Keywords: Monitoring; Spatialization; Exploration; Environment.

\section{Resumen}

Los conflictos ambientales se han convertido en una realidad global, en Brasil específicamente en el Estado de Pará, fue escenario de grandes historias tristes por buscar la preservación de los recursos naturales, presentando diversas categorías de conflictos, ya sea por la gran cantidad de materias primas o el Arco hace tala. El mapeo de estos conflictos es fundamental para la preservación de una calidad de vida saludable, por ello, este trabajo tiene como objetivo espacializar áreas de enfrentamientos ambientales en el Estado, tomando en cuenta los datos del Atlas de Justicia Ambiental, que contó con 24 ocurrencias para la área de estudio, sumada a los datos proporcionados por IBGE, así como el uso de software de procesamiento de datos Excel y ArcGIS. Es posible espacializar las ciudades de Pará con su tipo de conflicto. Por lo tanto, es posible afirmar la diversidad de conflictos que enfrenta el Estado, reflejando el desafío de atender y gestionar situaciones, de ahí que se recomiende ampliar este tipo de estudios, enfocándose en la gestión eficiente de los territorios.

Palabras clave: Vigilancia; Espacialización; Exploración; Medio ambiente.

\section{Introdução}

O Brasil é um país com grandes extensões territoriais e de uma biodiversidade incrível. O país é detentor de uma variedade de riquezas, seja de fauna, flora ou recursos minerais. Contudo, a grande disponibilidade de recursos naturais vem refletindo na quantidade de conflitos em decorrência desses bens. Em 2014, a Universidade Autônoma de Barcelona (UAB Barcelona) divulgou o Atlas de Justiça Ambiental do mundo, onde mapeou todos os conflitos ambientais, no qual, o Brasil apareceu em terceiro lugar com maior ocorrência de conflitos, em 2018, e passou para o segundo lugar em 2019.

No Atlas é possível encontrar o tipo de disputa e a região envolvida. Atualmente o Brasil se encontra na segunda posição do ranking, ficando atrás somente da Índia e encontra-se a frente de países como: Colômbia, Estados Unidos e China, principais causadores de poluição no mundo (WRI Brasil, 2019). A posição do Brasil no ranking reflete as ocorrências de conflitos. No estado do Pará especificamente, foi palco de grandes histórias entristecedoras, como a do seringueiro Chico Mendes e da ativista Dorothy Stang, assassinados em decorrência de conflitos por buscarem a preservação dos recursos naturais (Ebiografia, 2019; Estudo Pratico, 2018).

Em decorrência disso, o mapeamento das áreas com ocorrência de conflitos é fundamental para alertar a sociedade em geral da situação encontrada nos locais de disputa por recursos naturais, visando auxiliar com políticas públicas especificas para cada região. Este trabalho teve o intuito de espacializar as áreas de conflitos ambientais do estado do Pará, levando em consideração os dados do Atlas de Justiça Ambiental, da Universidade Autônoma de Barcelona (UAB Barcelona), que mapeou todos os conflitos em nível global e disponibilizou por meio de atlas, somando aos dados disponibilizados pelo senso do IBGE.

\section{Metodologia}

Este trabalho buscou realizar o estudo e mapeamento dos municípios do estado do Pará que apresentam algum tipo de disputa por recursos naturais, o que leva a conflitos ambientais. A área de estudo escolhida foi o Estado do Pará, localizada na região norte do país. O estado do Pará faz divisa com os estados do Amapá, Amazonas, Maranhão, Tocantins, Mato Grosso e Roraima onde a disponibilidade de recursos naturais é abundante (Figura 1). 
Figura 1: Mapa de localização área de estudo.

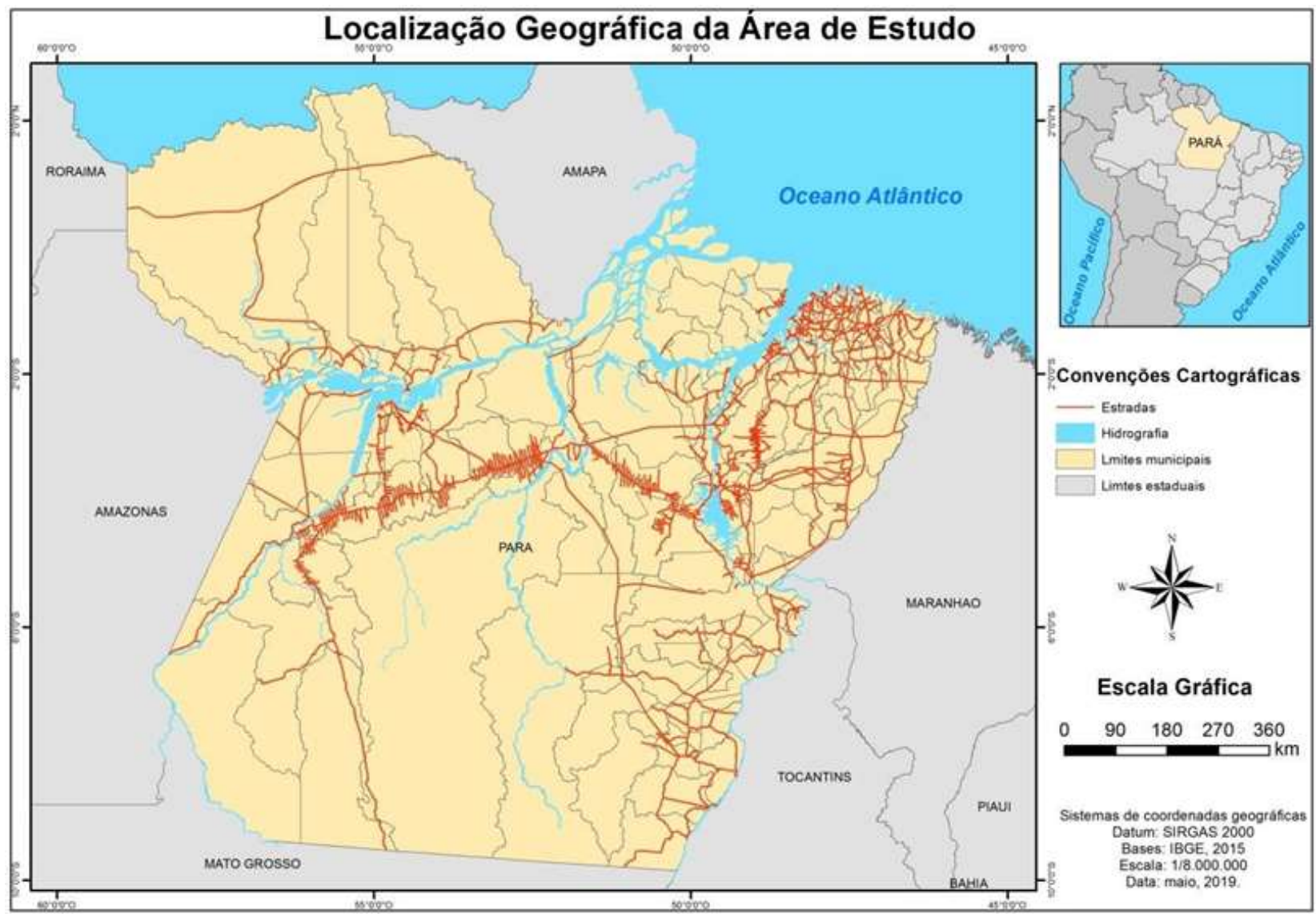

Fonte: Autores.

No estudo foi utilizado análise bibliográfica e mapeamento dos municípios do Estado do Pará que apresentam algum tipo de disputa por recursos naturais, levando a conflitos ambientais. Para este estudo foram utilizadas como base de dados principal o Atlas de Justiça Ambiental, da Universidade Autônoma de Barcelona (UAB Barcelona), assim como os dados do Instituto Brasileiro de Geografia e Estatística (IBGE), com os conflitos mapeados e os dados socioeconômicos de cada município que apresentou disputa por recursos naturais.

Para execução do trabalho, foram utilizados o software Excel para edição de atributos e o software ArcGIS para processamento de dados shapefiles das áreas de estudo, as bases cartográficas foram provenientes do IBGE, a partir disso, foi realizado o recorte somente das áreas de interesse e assim plotagem das mesmas, através do mapa de localização das regiões com os respectivos conflitos.

\section{Resultados e Discussão}

De acordo com o levantamento, no cenário mundial, o Brasil é o segundo país com maior quantidade de registros de conflitos ambientais, com cerca de 130 conflitos (Figura 2), classificados como: nuclear, mineração, gestão de resíduos, gerência de água, biomassa, combustíveis fosseis, conflitos terrestres, recreação turística, conflitos de conservação da biodiversidade e conflitos industriais. 
Figura 2: Gráfico da ocorrência de conflitos por países.

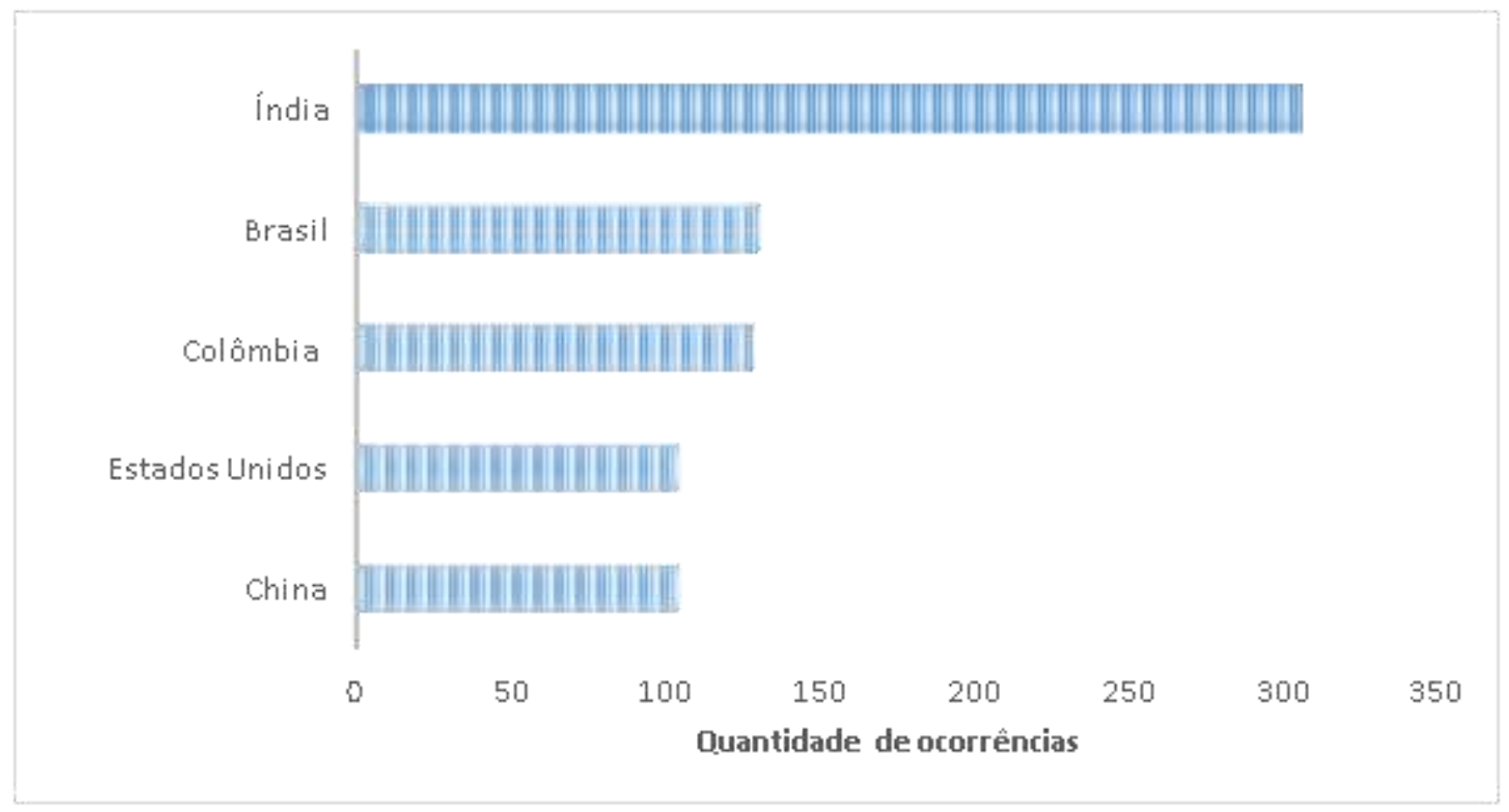

Fonte: Autores.

A partir da análise dos dados, foi possível listar os municípios que apresentaram conflitos ambientais, assim como a respectiva categoria de conflitos. O Pará apresenta 24 conflitos registrados, no qual, tem mais disputa por biomassa, em detrimento da extensão de mata amazônica que o Estado possui, somado aos projetos agroindustriais implantados, no entanto, o desenvolvimento da indústria acarreta conflitos desde a extração de matéria-prima até a energia para a produção dos materiais beneficiados, por isso existe um grande desacordo de ideias sobre a implantação de hidrelétricas. A seguir está a lista de municípios que apresentaram conflitos:

•Conflitos Industriais e Utilitários: Barcarena e Belém;

-Conflitos Terrestres e de Biomassa: Anajás, Anapu, Cachoeira do Arari, Jacundá, Novo Progresso, Prainha, Porto de Moz, Santarém, Tomé-Açu e Xinguara;

-Conflitos pela Gerencia de Água: Itaituba, Marabá, Tucuruí e Vitória do Xingu;

-Mineração Mineral e Extração de Materiais de Construção: Canaã dos Carajás, Jacareacanga, Juruti, Moju, Oriximiná, Ourilândia do Norte, Senador José Porfírio e São Félix do Xingu.

Além da tabulação, produziu-se o produto cartográfico com os conflitos na distribuição espacial do Estado do Pará (figura 3). 
Figura 3: Munícipios e seus tipos de conflitos.

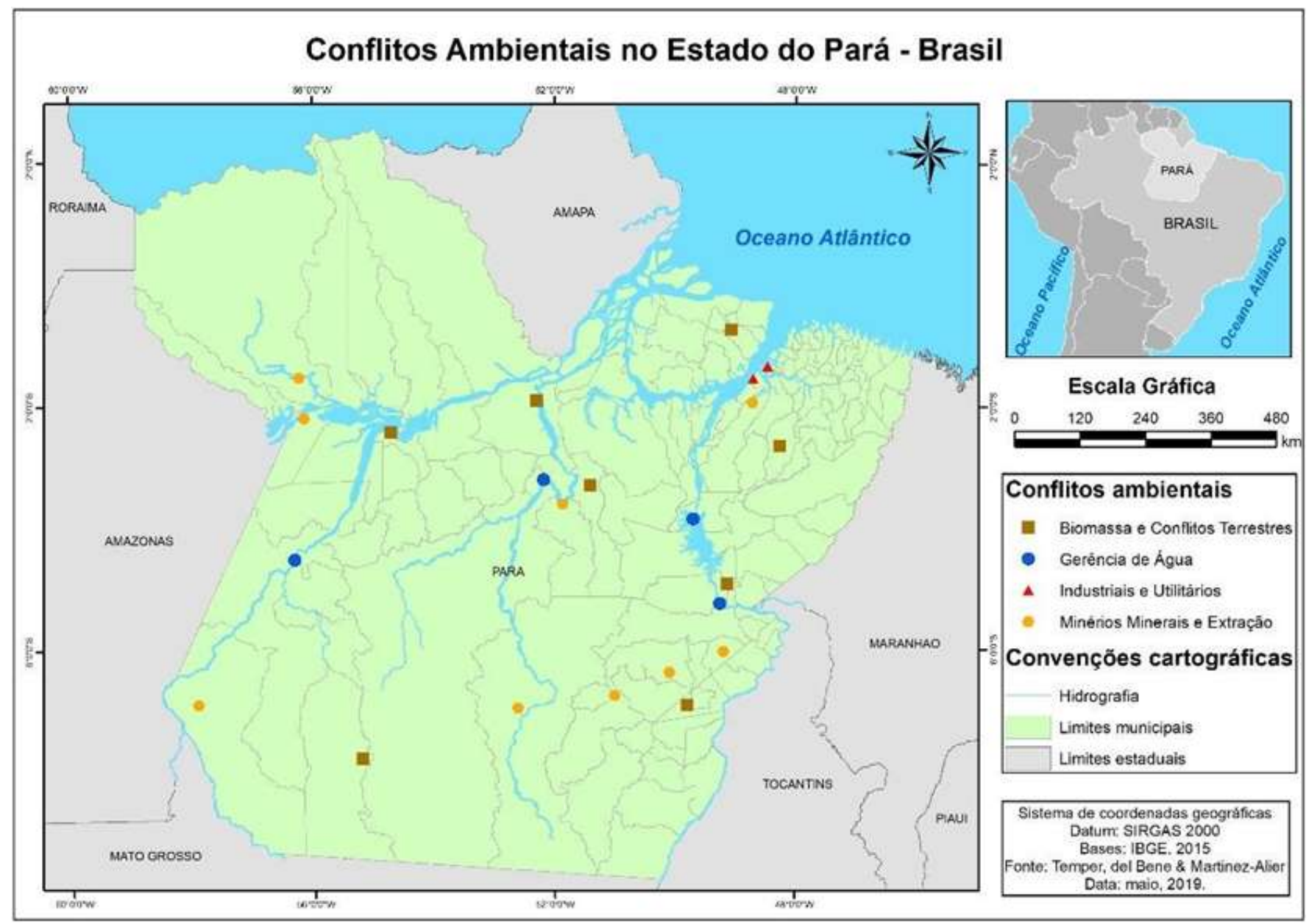

Fonte: Autores.

Analisando o mapa acima de acordo com a legenda descrita no mesmo, pode-se observar o primeiro destaque, os enfrentamentos causados por biomassa e conflitos terrestres, que se originou do desenvolvimento e aprimoramento de novas formas de obtenção de energia limpa e de fato é obtido vantagens. Porém podem causar impactos negativos, sendo alguns deles, a necessidade de grandes plantações para o desenvolvimento de sua matéria prima, por isso a desflorestação e a destruição de habitats naturais, devem ser considerada e realizada com muito cuidado, contaminação do solo e de mananciais pelo uso de agrotóxicos, a destruição do solo pela erosão, a poluição proveniente da queima da biomassa e dentre outros.

Segundo o Instituto Nacional de Pesquisas Espaciais - INPE (2019), a quantificação da biomassa e do carbono do bioma Amazônia é objeto de estudos de diversos pesquisadores que, a partir de diferentes metodologias e dados, buscam entender a dinâmica da vegetação da região (Chave et al.,2005, Mazzei et al.,2010, West\&Putz, 2014, Sist et al 2014, Carneiro et al 2020). Com o intuito de melhoria de métodos, mapas desenvolvidos a partir desses estudos e podem ser utilizados como base de ferramentas de políticas públicas e gestão socioambiental.

A situação de gerência de água, como visualizado acima, encontram-se em 4 (quatro) regiões. A captação de água, lançamento de efluente decorrentes das atividades, por exemplos as indústrias, e carência de planejamento hídrico, causam conflitos. Problemática relacionada à grande explosão demográfica de cada região, prejudicando a população, caso não haja um gerenciamento desse recurso natural.

A gestão das águas eficiente deve ser constituída por uma política, que estabeleça as diretrizes gerais do planejamento do uso, controle e proteção das águas. Para o benefício do meio ambiente e do homem, é necessário buscar soluções, como o 
uso da geotecnologia, para essas disputas ou amenizar possíveis prejuízos que a população local poderá sofrer caso fiquem impossibilitadas de utilizar a riqueza hídrica do município (Ferreira, 2015).

Um conflito que atinge uma quantidade maior de pessoas ocorre na cidade de Belém, capital do Pará, que foi classificado como "Conflitos industriais e utilitários". O problema é a produção de couro no distrito de Icoaraci, área urbana populosa que atinge cerca de 130 mil pessoas, que iniciou em 2005 e está operante atualmente e que traz problemas a saúde por meio de poluição do ar, contaminação do solo e da água.

Conforme a pesquisa, "A mobilização das comunidades e os estudos científicos concluíram que há um impacto dos curtumes no meio ambiente e na saúde humana [...]" e "Um dos estudos de pesquisadores mostra que um dos elementos tóxicos utilizados no processo de preparação do couro é o cromo, que é irritante, afetando o trato respiratório superior, obstrução de dutos de ar e aumento das chances de desenvolver câncer de pulmão, nasal ou sinusal (Germann, H. P. (1995), Paiva \& Morisso (2009). Estão associadas à asma, bronquite, pólipos do trato respiratório superior e faringite" (Silva et al., 2018).

A mineração mineral possui maior destaque no Estado Pará devido ao crescimento demográfico nos municípios da Amazônia, influenciado por atividades de exploração mineral ou induzidas por elas nestes espaços geográficos (Agência Nacional de Mineração, 2019). Consequentemente, impulsionaram e modificaram a funcionalidade dos municípios sedes da exploração mineral, induzindo novas dinâmicas territoriais, com reflexos demográficos, financeiros, ambientais, sociais, políticos e ampliando os conflitos (Palheta et al., 2017, Santos, 2018, Congilio, 2019).

Segundo Palheta et al. (2017), é fundamental analisar o peso que questões ambientais, políticas, culturais e sociais têm na balança para se debater o futuro da atividade na região. Ainda segundo o autor a experiência paraense tem demonstrado que a cada nova jazida de minério descoberta, novas formas de conflito se instalam, criando em escala local conflitos entre a exploração do subsolo e a reprodução de territórios tradicionais, conflitos entre a exploração do subsolo e as dinâmicas territoriais anteriores e, por fim, conflitos entre a exploração do subsolo e o ecossistema.

É importante ressaltar que um dos conflitos ambientais está localizado no Rio Xingu, local da instalação das obras da usina de Belo Monte iniciada em 2005 e que será projetada para gerar cerca de 11.233 megawatts. Entretanto, os impactos ambientais serão devastadores, pois refletirá diretamente no cenário socioambiental, tendo problemas como: desmatamento, perda de biodiversidade, distúrbio em potencial dos sistemas hidrológicos, geológicos e ecológicos, poluição do ar, insegurança alimentar, problemas de saúde, entre outros, afetando diretamente a população ao redor (cerca de 20 mil pessoas) de forma a diminuir a produção agrícola e de pesca para o mercado e para subsistência que por sua vez, altera significativamente a economia local, obrigando aqueles que não foram afetados diretamente a procurar outros meios e/ou locais para viver (Fleury \& Almeida, 2013, Comegna,2016, Ramos \&Alves, 2018).

Destaca-se também os conflitos de interesses por parte dos grupos indígenas como os Juruna, Xikrin, Arara, Xipaia, Kuruaya e Kayapó que vão ser diretamente afetados, já que não abrem mão de seu território e terão que ser obrigados a ser remanejados. Por isso, diversas mobilizações por parte destes ocorrem frequentemente, como bloqueios, protestos, ideias alternativas e a idealização peculiar de defesa a mãe natureza, além de buscarem meios legais para respaldo e impedimento do seguimento das obras, sempre apoiados por movimentos sociais, grupos religiosos e afins. Entretanto, por resistência das partes que têm interesse em seguir com as obras da hidrelétrica de Belo Monte (respaldados pela justiça), os ativistas tomam medidas mais radicais como ocupação das obras e conflitos armados que estabelece a condição de criminalização dos mesmos.

\section{Considerações Finais}

Em decorrência de tudo que foi exposto neste trabalho, conclui-se que o crescimento das indústrias que exploram recursos naturais afeta a biodiversidade, seja em pequena ou larga escala. Apesar dos estudos socioambientais, problemas de 
saúde, pressão para que os órgãos regulamentadores atuem de forma mais eficaz e decisões judiciais, os conflitos seguem de forma acintosa pelo fato de que as indústrias e afins ainda estejam operando de maneira a prejudicar as pessoas e o meio ambiente. É necessária a conscientização por parte das indústrias e buscar outros meios de realizar suas atividades sem que prejudique os seres vivos que ali habitam.

\section{Referências}

Agência Nacional de Mineração. (2019) Informe Mineral Estado Do Pará 2018. Belém - PA, maio. https://www.gov.br/anm/pt-br/centrais-deconteudo/publicacoes/serie-estatisticas-e-economia-mineral/informe-mineral/publicacoes-regionais/informe_mineral_para_2018.pdf.

Carneiro, F. S., Ruschel, A. R., Oliveira. F. A., Soares, M. H. M., Pinto, M. V. P., Rodrigues, C. F. A. (2020). Resilience of Above-ground Biomass in Experimental Areas in the Eastern Brazilian Amazon. Biodiversidade Brasileira, 10(3): 74-83. DOI: 10.37002/biobrasil.v10i3.1660.

Celia Regina Congilio, Rosemayre Bezerra, Fernando Michelotti . (2019). Mineração, trabalho e conflitos amazônicos no sudeste do Pará [livro eletrônico]. I organizadores do volume. -1.ed.-Marabá, PA : iGuana, 2019. 1 livro digital.—(Mineração: trabalho, territórios e conflitos na região de Carajás ; v.1).

Chave, J., Andalo, C., Brown, S. et al. (2005)Tree allometry and improved estimation of carbon stocks and balance in tropical forests. Oecologia.145: 87. https://doi.org/10.1007/s00442-005-0100-x.

Comegna, M. A. (2016). Usina Hidrelétrica De Belo Monte E Justiça Ambiental. XVIII encontro Nacional de geógrafos, Anais. http://www.eng2016.agb.org.br/resources/anais/7/1467226921_ARQUIVO_UsinadeBeloMonteeJusticaAmbiental2016.pdf.

Ebiografia. (2019). Biografia de Chico Mendes. https://www.ebiografia.com/chico_mendes/.

Estudo Pratico. Biografia de Dorothy Stang. https://www.estudopratico.com.br/quem-foi-dorothy-stang/.

Ferreira, D. L. N. Conflito pelo uso da água na Amazônia Brasileira: Uma análise envolvendo a atividade Mínero-Metalúrgica a as comunidades Ilha São João e Curuperé no município de Barcarena-PA. Dissertação de mestrado. Instituto de Filosofia e Ciências Humanas. Universidade Federal do Pará (UFPA), 2015 .

Fleury, L. C. \& Almeida, J. (2013). A construção da Usina Hidrelétrica de Belo Monte: conflito ambiental e o dilema do desenvolvimento. Ambiente \& Sociedade [online]. 2013, v. 16, n.4 , pp. 141-156. <https://doi.org/10.1590/S1414-753X2013000400009>. Epub 14 Fev 2014. ISSN 1809-4422. https://doi.org/10.1590/S1414-753X2013000400009.

Germann, H. P. (1995). Chrome Tannage from the Viewpoint of Ecology. Journal of Society Leather Technology Centre, v. 79 , p. 82-85.

Instituto Nacional De Pesquisas Espaciais - INPE. EBA - Estimativa de Biomassa Na Amazônia. Melhoria dos métodos de estimativa de biomassa e de modelos de estimativa de emissões por mudança de uso da terra. http://www.ccst.inpe.br/projetos/eba-estimativa-de-biomassa-na-amazonia/.

Mazzei, L., Sist, P., Ruschel, A.R., Putz F.E., Marco P., Pena, W., \& Ferreira J.E.R. (2010). Above-ground biomass dynamics after reduced-impact logging in the Eastern Amazon. Forest Ecology and Management 259: 367-373. https:// doi.org/10.1016/j.foreco.2009.10.031.

Paiva, R. M. M. \& Morisso, F. D. P. (2009). Uma breve revisão sobre processos de curtimentos alternativos e considerações sobre a aplicação de cálculos químicos na previsão de propriedades desses sistemas. Revista Tecnologia e Tendências. P89-96.

Palheta, J. M., Silva, C. N., Oliveira Neto, A., Nascimento, F. R. (2017). Conflitos pelo uso do território

na Amazônia mineral. Mercator (Fortaleza) [online]. 2017, v. 16. http://www.mercator.ufc.br/mercator/article/view/2100. V. 16, ed.16023.

Ramos, A. M., Alves. H. P. F. ( 2018). Conflito socioeconômico e ambiental ao redor da construção da Usina Hidrelétrica Belo Monte. Desenvolvimento e Meio Ambiente, v. 46, p. 174-196, agosto. DOI: 10.5380/dma.v46i0.50248. e-ISSN 2176-9109.

Santos, A. A. S. (2018) Mineração e conflitos fundiários no sudeste paraense. Dissertação (Mestrado) - Universidade Federal do Sul e Sudeste do Pará, Campus Universitário de Marabá, Programa de Pós-Graduação em Dinâmicas Territoriais e Sociedade na Amazônia, Marabá, 2018.

Silva, A. L. S. et al. Impactos ambientais gerados por efluentes de curtume em meio aquático e na saúde humana o caso de uma indústria no distrito industrial de Icoaraci em Belém do Pará. http://www.sbpcnet.org.br/livro/64ra/resumos/resumos/3564.htm.

Sist, P., Mazzei, L., Blanc, L. \& Rutishauser, E. (2014). Large trees as key elements of carbon storage anddynamics after selective logging in the Eastern Amazon. Forest Ecology and Management. 318: 103-109. Doi:10.1016/j.foreco.2014.01.005.

Temper, L., Del Bene, D. \& Martinez-Alier, J. (2019). Mapeando as fronteiras e linhas de frente da justiça ambiental global: o EJAtlas. Journal of Political Ecology 22: 255-278. 2015. https://ejatlas.org/about.

West, T.A.P., Vidal, E. \& Putz, F.E. (2014). Forest biomass recovery after conventional and reduced-impact logging in Amazonian Brazil. Forest Ecology and Management, 314: 59-63. https://doi.org/10.1016/j. Foreco. 2013.11.02.

WRI Brasil.(2019). Os países que mais emitiram gases de efeito estufa nos últimos 165 anos. https://wribrasil.org.br/pt/blog/2019/04/ranking-paises-que-maisemitem- carbono-gases-de-efeito-estufa-aquecimento-global. 